\title{
Stochastic Evaluation of Compound Indoor Electrification Environment Based on Mutual Intermediation between Different Fields
}

\author{
Hitoshi Ogawa ${ }^{\dagger}$, Mitsuo Ohta ${ }^{\dagger \dagger}$ and Hirofumi Iwashige ${ }^{\dagger \dagger \dagger}$ \\ ${ }^{\dagger}$ Facul. of Management and Information Systems, Prefectural University of Hiroshima \\ 1-1-71 Ujina-Higashi, Minami-ku, Hiroshima 734-8558, Japan \\ ${ }^{\dagger \dagger}$ Emeritus Professor, Hiroshima University \\ 1106, 1-7-10 Matoba, Minami-Ku, Hiroshima 732-0824, Japan \\ ${ }^{\dagger \dagger}$ Graduate School of Education, Hiroshima University \\ 1-1-1 Kagamiyama, Higashi-Hiroshima 739-8524, Japan
}

E-mail: ${ }^{\dagger}$ hogawa@hirojo-u.ac.jp, ${ }^{\dagger \dagger}{ }^{\dagger \dagger}$ ohta-3322@mdd.spacetown.ne.jp,
${ }^{\dagger}$ hiroiwa@hiroshima-u.ac.jp

\begin{abstract}
In recent years, according to very rapid increase of various kinds of information and communication equipment like personal computers and portable radio transmitters, not only a problem of the technical characteristics of equipment and propagation but also a problem of the influence to the environment including human body has become gradually important. In this study, some evaluation and/or measurement methods for compound effect based on the inter-subjective relationships between sound (with other environmental factors) and psychological or physiological factors have been proposed, especially through some examples of the physical factors (sound, lights, magnetic field and temperature) and the psychological or physiological factors (noise evaluation values, mean blood pressure and pulsation) surrounding an indoor electrification environment. Finally, with an application to actually measured data, a part of the effectiveness of the proposed method has been also experimentally confirmed.
\end{abstract}

\section{Introduction}

Needless to say, our living circumstance is surrounded by various kinds of wave environmental factors, such as sound, light, EM waves and so on. Besides, they form indivisible problematique in a complex manner by the natural, social and human factors. And, in these wave motional environmental problems including sound, light and EM waves, the studies on compound and/or accumulation effects among those wave environmental factors are becoming more important year by year, according to the increase of various types of information and communication systems such as personal computers and portable radio transmitters. Furthermore, these kinds of problems are studied more widely not only on the EM environment itself leaked by an electronic equipment, but also on the biological effects in the living environment [1]. For instance, concerning their individual and/or compound effects on a living body, it is well-known that there are many unsolved questions like video (or visual) display terminal (VDT) symptoms. To cite several concrete examples, our brain nerve is well-known to be mostly influenced below the frequency of $20 \mathrm{~Hz}$ at $\mathrm{EM}$, sound and light waves. This is recognized in an amplitude modulation of the high frequency domain as well. Also, the generating order, the interval and each duration of flashes and noises along a time axis produce some problems for the relationships to physiological processes. Otherwise, there are more other similar problems, such as a predominant effect of sight (Hearing seems to be dragged to sight having higher warning ability.), a promotion effect among different kind of senses, participation to VDT symptom groups (e.g. complain of general malaise) as well as multiplication effect with stress, relationships between daily rhythm of human life and the effects to a pineal body by EM field including light, some changes of brain waves by stimulus of light and sound, and so on. In these studies, it is generally pointed out that the first important topic is to find some new measurement and evaluation methods even in a quantitative approximation.

In general, it is well-known that usually the EM and sound waves are precisely measured in a frequency domain under the standardized measuring situation in a reverberation room, anechoic room and radiofrequency anechoic chamber. Surely, these standard methods in a frequency domain are useful especially for the purpose of analyzing (from the separatism-first viewpoint) the mechanism of individual phenomena, but they seem to be insufficient for evaluating (from the relationism-first viewpoint) total images on the compound or mutual relationship among sound, light and EM waves in the actual complicated living circumstances [2]. In previously published papers [3-9], for a trial, we have proposed an extended regression analysis method [10] reflecting 
various type latent correlation information of not only the ordinary lower orders, but also of the higher orders in order to quantitatively investigate and evaluate the mutual relationship among them, especially from a viewpoint of "Relationism-first".

In this paper, in relation to the previously published methodological studies [11-15], a stochastic methodology to grasp the mutual relationship between physical factors (sound, lights, magnetic field and temperature) and psychological or physiological factors (noise evaluation values, mean blood pressure and pulsation) in an indoor acoustic environment is discussed, especially through a system model from the extended regression analysis. Only after such a relational analysis, in order to mutually predict their probabilistic behavior from one to another, we have proposed a parameter estimation method based on an internal statistical architecture of the joint probability among these factors. Then, the validity and effectiveness of our proposed method are confirmed through some principle experiment by an application to the observation data in the room of an actual living environment.

\section{General Theory}

In order to evaluate quantitatively and hierarchically the complicated relationship between two variables (e.g. sound and noise evaluation value), let us introduce first a generalized regression analysis method [10] employing not only the linear correlation but also the nonlinear correlation information among them. Especially, in the case with a prediction variable $x$ and a criterion variable $y$, it must be noticed that the whole information on mutual correlation between them is included in the conditional probability distribution $P(y \mid x)$ :

$$
\begin{aligned}
& P(y \mid x)=\frac{P(x, y)}{P(x)}=\frac{P_{0}(y) \sum_{m=0}^{\infty} \sum_{n=0}^{\infty} A_{m n} \varphi_{m}^{(1)}(x) \varphi_{n}^{(2)}(y)}{\sum_{m=0}^{\infty} A_{m 0} \varphi_{m}^{(1)}(x)}, \\
& A_{m n} \equiv\left\langle\varphi_{m}^{(1)}(x) \varphi_{n}^{(2)}(y)\right\rangle_{x, y},
\end{aligned}
$$

where $P_{0}(y)$ denotes the fundamental probability distribution of $y \cdot \varphi_{m}^{(1)}(x)$ and $\varphi_{n}^{(2)}(y)$ are orthonormal polynomials with two weighting functions $P_{0}(x)$ and $P_{0}(y)$, respectively. $P_{0}(x)$ also denotes the fundamental probability distribution of $x .\langle\cdot\rangle$ denotes the averaging operation with respect to the random variables. Thus, the information on the various types of linear and nonlinear correlations between $x$ and $y$ is reflected hierarchically in each expansion coefficient $A_{m n}$ [16].

After employing an orthogonal series expansion expression of $y$ appearing in Eq. (3) and the orthonormal condition of $\varphi_{n}^{(2)}(y)$, the regression function as a typical regression relationship between $x$ and $y$ can be explicitly given in Eq. (4), as follows:

$$
\begin{aligned}
& y=\sum_{j=0}^{1} C_{1 j} \varphi_{j}^{(2)}(y), \\
& \langle y \mid x\rangle=\frac{\sum_{m=0}^{\infty} \sum_{n=0}^{1} C_{1 n} A_{m n} \varphi_{m}^{(1)}(x)}{\sum_{m=0}^{\infty} A_{m 0} \varphi_{m}^{(1)}(x)},
\end{aligned}
$$

where the expansion coefficients $C_{10}$ and $C_{11}$ are calculated in advance through the realization of the orthonormal polynomial $\varphi_{n}^{(2)}(y)$. Thus, after estimating the expansion coefficient $A_{m n}$ defined by Eq. (2) on the basis of the observed data on $x$ and $y$, the regression function between $x$ and $y$ can be evaluated.

Furthermore, a specific probability distribution $P_{s}(y)$ of $y$ based on an arbitrary type random fluctuation of regressively related stochastic variable $x$ can be predicted by an averaging operation based on Eq. (1), as follows:

$$
\begin{aligned}
& P_{s}(y) \equiv\langle P(y \mid x)\rangle_{x}=P_{0}(y) \sum_{n=0}^{\infty} B_{n} \varphi_{n}^{(2)}(y), \\
& B_{n}=\left\langle\frac{\sum_{m=0}^{\infty} A_{m n} \varphi_{m}^{(1)}(x)}{\sum_{m=0}^{\infty} A_{m 0} \varphi_{m}^{(1)}(x)}\right\rangle_{x} .
\end{aligned}
$$

Concretely, the well-known normal distribution can be practically introduced as two fundamental probability distributions $P_{0}(x)$ and $P_{0}(y)$. By utilizing Hermite polynomials: $\mathrm{H}_{i}(\cdot)$, the regression function $\langle y \mid x\rangle$ in Eq. (4) and the regression parameter $A_{m n}$ in Eq. (2) can be realized respectively, as follows:

$$
\begin{aligned}
& \langle y \mid x\rangle=\mu_{y}+\sigma_{y} \frac{\sum_{m=0}^{\infty} A_{m 1} \frac{1}{\sqrt{m !}} \mathrm{H}_{m}\left(\frac{x-\mu_{x}}{\sigma_{x}}\right)}{\sum_{m=0}^{\infty} A_{m 0} \frac{1}{\sqrt{m !}} \mathrm{H}_{m}\left(\frac{x-\mu_{x}}{\sigma_{x}}\right)}, \\
& A_{m n} \equiv\left\langle\frac{1}{\sqrt{m !}} \mathrm{H}_{m}\left(\frac{x-\mu_{x}}{\sigma_{x}}\right) \frac{1}{\sqrt{n !}} \mathrm{H}_{n}\left(\frac{y-\mu_{y}}{\sigma_{y}}\right)\right\rangle_{x, y} .
\end{aligned}
$$

Furthermore, specific probability distribution $P_{s}(y)$ in Eq. (5) and the parameter $B_{n}$ in Eq. (6) can be finally realized respectively, as follows:

$$
P_{s}(y)=\frac{1}{\sqrt{2 \pi} \sigma_{y}} e^{-\frac{\left(y-\mu_{y}\right)^{2}}{2 \sigma_{y}^{2}}} \sum_{n=0}^{\infty} B_{n} \frac{1}{\sqrt{n !}} \mathrm{H}_{n}\left(\frac{y-\mu_{y}}{\sigma_{y}}\right),
$$




$$
B_{n}=\left\langle\frac{\sum_{m=0}^{\infty} A_{m n} \frac{1}{\sqrt{m !}} \mathrm{H}_{m}\left(\frac{x-\mu_{x}}{\sigma_{x}}\right)}{\sum_{m=0}^{\infty} A_{m 0} \frac{1}{\sqrt{m !}} \mathrm{H}_{m}\left(\frac{x-\mu_{x}}{\sigma_{x}}\right)}\right\rangle .
$$

\section{Experiment}

In order to confirm a part of the effectiveness of the proposed method, two kinds of indoor acoustic environment have been chosen as a specific electrification environment. The former experiment is carried out for analyzing the human psychological characteristics (noise evaluation value) against the physical stimuli (sound, light and room temperature). The latter one is carried out for analyzing the human physiological characteristics (mean blood pressure and pulsation) against the physical stimuli (sound and magnetic field).

\subsection{Extended Regression Analysis between Physical Stimuli and Psychological Evaluation}

This experiment is carried out in our climate chamber. The room is illuminated by twelve sets of white incandescent ceiling lamps. A white noise is fed into the chamber by loudspeakers. The temperature of the chamber is controlled by heat pump type air conditioners. Environmental factors for experiment are chosen under the actual conditions of daily life. Four categories of thermal exposure, four categories of noise level, five categories of illuminance exposure are used. Magnitude Estimation Method without standard value is employed to obtain the evaluation value for many complex living conditions. All subjects were healthy young men and women.

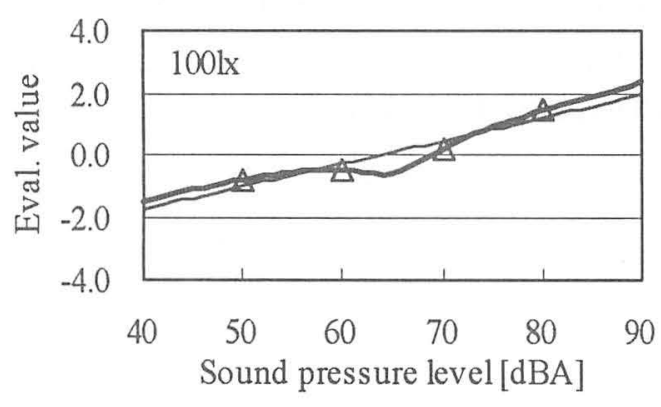

(a) $M=11$

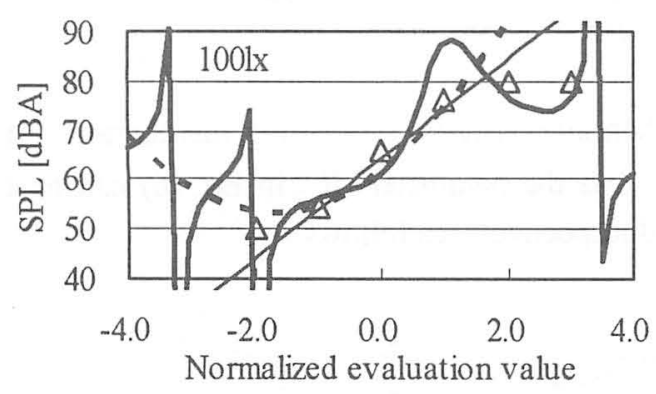

(b) $M=5$

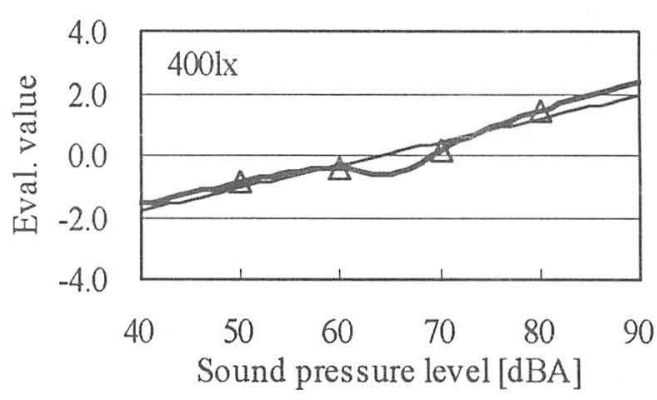

(c) $M=11$

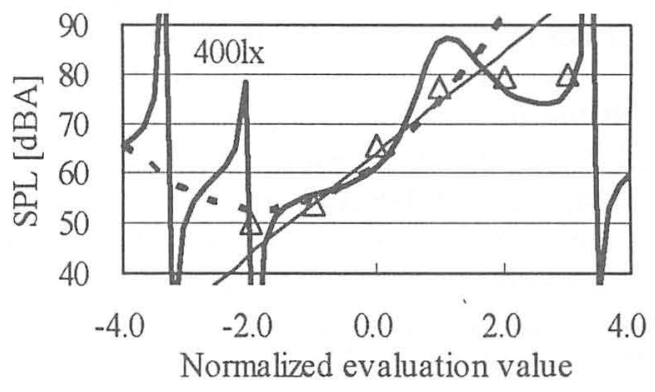

(d) $M=5$

Fig.1 Comparisons between experimentally sampled conditional mean values $(\Delta)$ and theoretical regression curves ( - : linear, - : higher order approximation) for the regression of noise stimulus and noise evaluation value at $25^{\circ} \mathrm{C}$.

Figure 1 shows comparisons between experimentally sampled conditional mean values and theoretical regression curves for the regression of noise stimulus and noise evaluation value at $25^{\circ} \mathrm{C}$. In these figures, parameter $M$ denotes the truncate term of the expanded expression of the regression function $\langle y \mid x\rangle$ in Eq. (7). In figures 1(a) and 1(c), it is shown the conditional mean values of normalized noise evaluation value for sound pressure level change. In figures 1(b) and 1(d), it is also shown the conditional mean values of sound pressure level for normalized noise evaluation value change in reverse. In these figures, the theoretical regression curves explain the tendency of conditional mean value by higher order approximation well.

\subsection{Extended Regression Analysis between} Physical Stimuli and Physiological Responses and mutual estimation of probability distribution

The proposed method is applied to the measured data using a VDT equipped with a cathode-ray tube (CRT) type display while playing a video game (a kind of flight simulators) in living room environment. In this paper, only an essential point is described here. The sound pressure level [dBA], the r.m.s value of the magnetic field strength $[\mathrm{mG}]$, the mean blood pressure and pulsation are simultaneously measured. All subjects were healthy young women. 


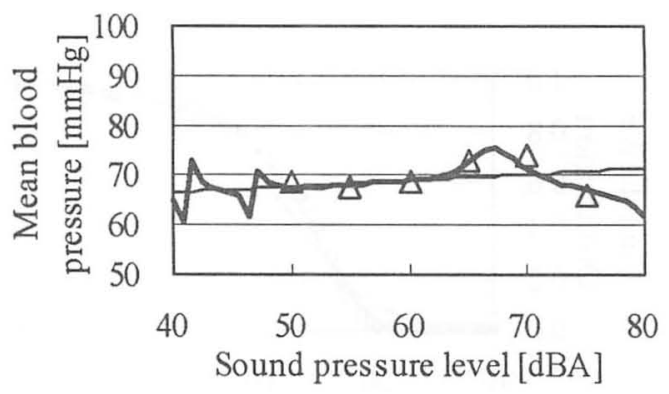

(a) $M=10$

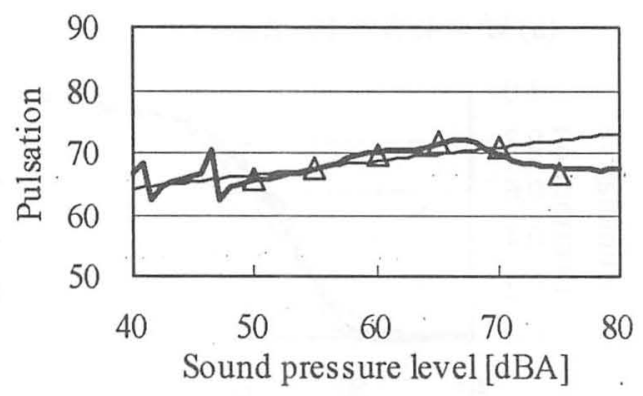

(b) $M=10$

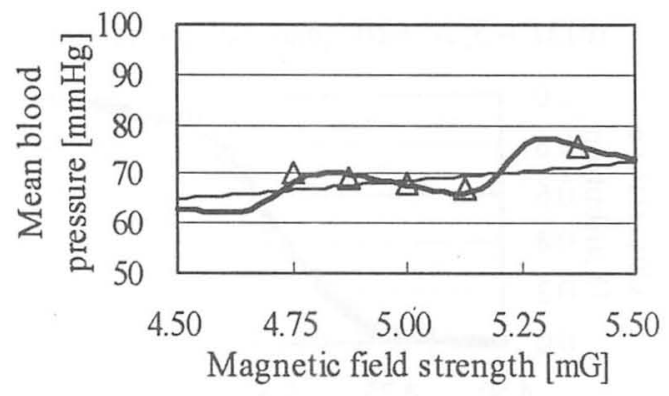

(c) $M=6$

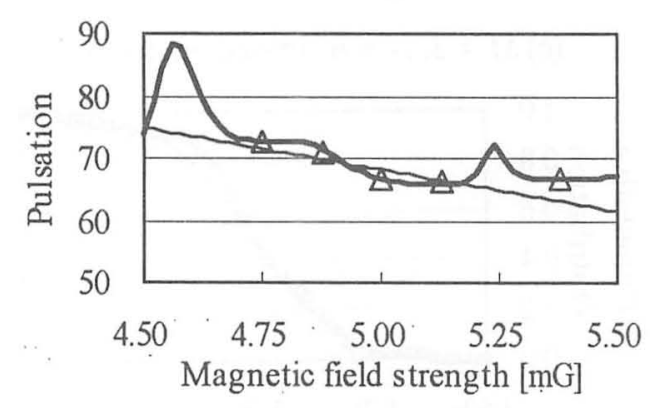

(d) $M=11$

Fig.2 Comparisons between experimentally sampled conditional mean values $(\Delta)$ and theoretical regression curves ( - : linear, $-\longrightarrow$ : higher order approximation) from physical factors to physiological factors.

Figure 2 shows comparisons between experimentally sampled conditional mean values and theoretical regression curves from physical factors (i.e. sound and magnetic field) to physiological factors (i.e. mean blood pressure and pulsation). In these figures, the theoretical regression curves explain the actual tendency roughly well even in the form of conditional mean and furthermore higher order approximation. In addition to this, the effect of the magnetic field to the pulsation appeared in figure 2(d) seems quite different from the others.

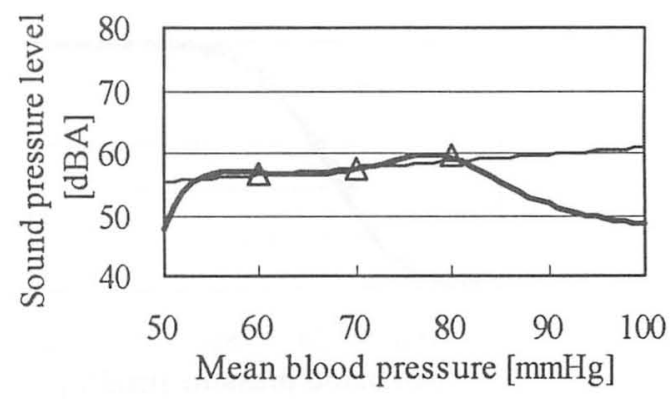

(a) $M=4$

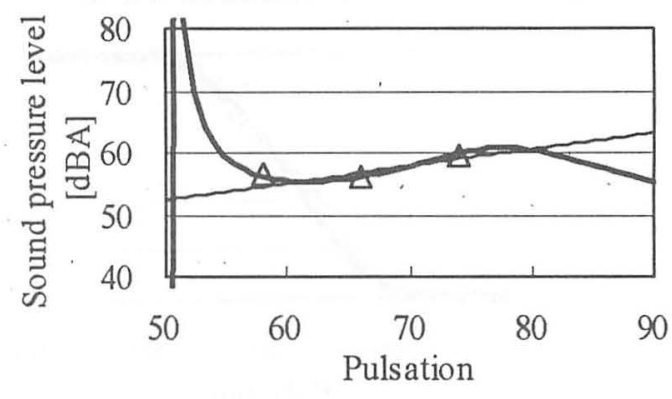

(b) $M=3$

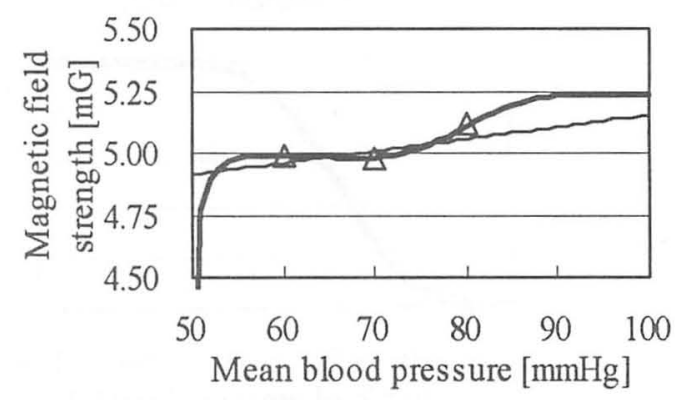

(c) $M=3$

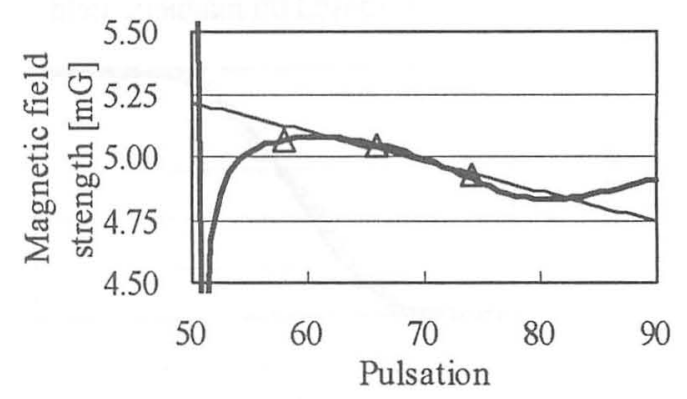

(d) $M=3$

Fig.3 Comparisons between experimentally sampled conditional mean values $(\Delta)$ and theoretical regression curves ( - : linear, $\_$: higher order approximation) from physiological factors to physical factors.

Figure 3 shows comparisons between experimentally sampled conditional mean values and theoretical regression curves from physiological factors (i.e. mean blood pressure and pulsation) to physical factors (i.e. sound and magnetic field) in reverse. In these figures, the theoretical regression curves also explain the tendency of conditional mean value by higher order approximation well. Furthermore, the expanded expression of the regression 
function in figure 3 requires fewer truncate term $M$ than those of the regression function in figure 2.

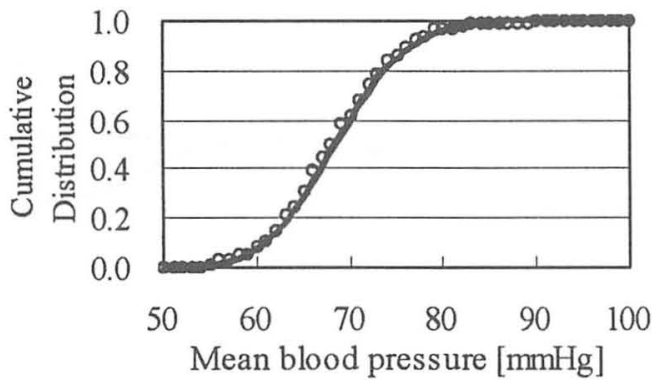

(a) $M=10, N=4$ (based on sound)

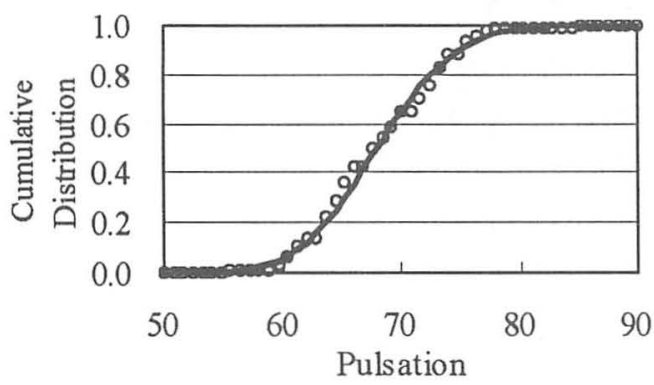

(b) $M=10, N=3$ (based on sound)

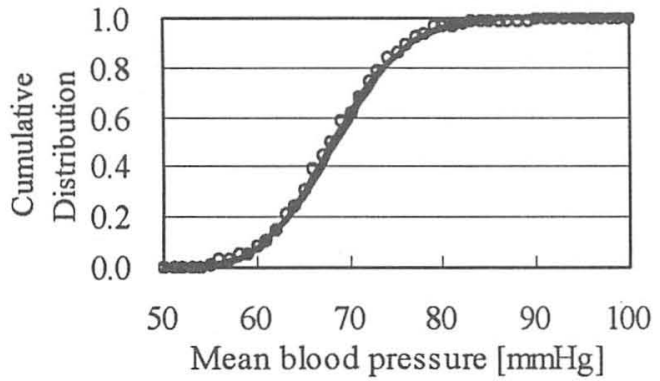

(c) $M=6, N=3$ (based on magnetic field)

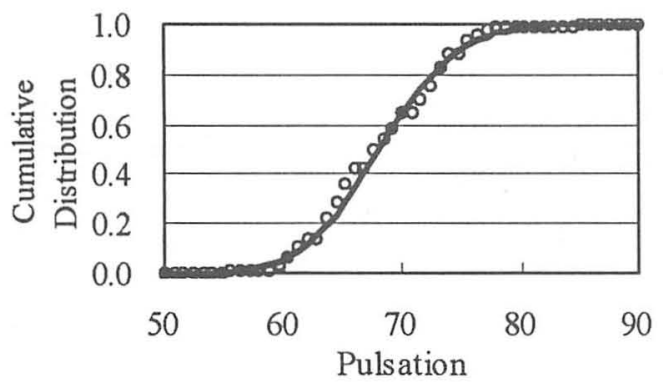

(d) $M=11, N=3$ (based on magnetic field)

Fig.4 A comparison between experimentally sampled specific probability distribution $(0)$ and theoretically estimated distribution curves ( - : initial term, - : higher order approximation) from physical factors to physiological factors.

Figure 4 shows comparisons between experimentally sampled specific probability distribution $P_{s}(y)$ and theoretically estimated distribution curves from physical factors (i.e. sound and magnetic field) to physiological factors (i.e. mean blood pressure and pulsation) by use of
Eqs. (9) and (10).

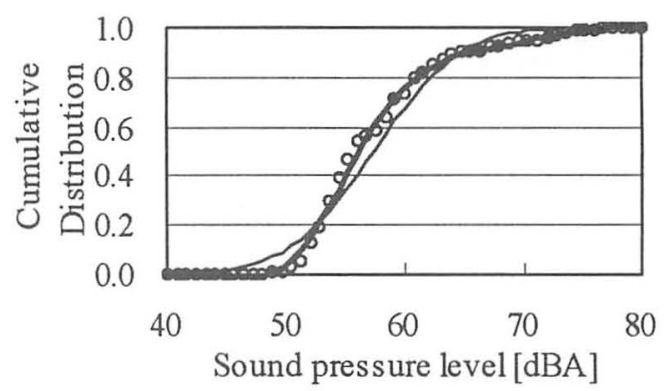

(a) $M=4, N=10$ (based on m.b.p.)

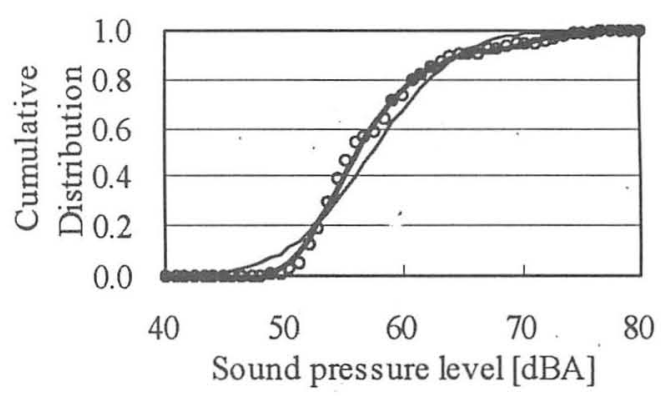

(b) $M=3, N=10$ (based on pulsation)

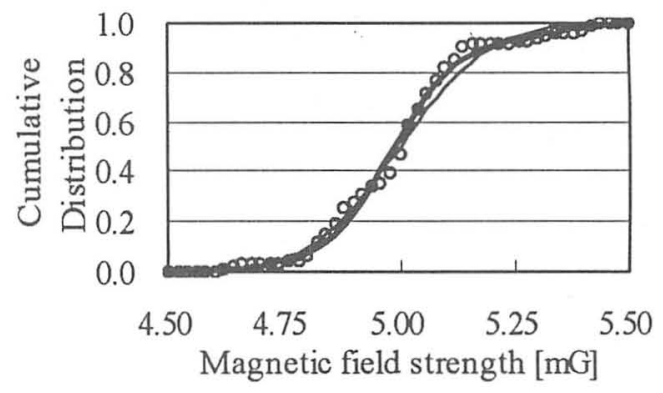

(c) $M=3, N=6$ (based on m.b.p.)

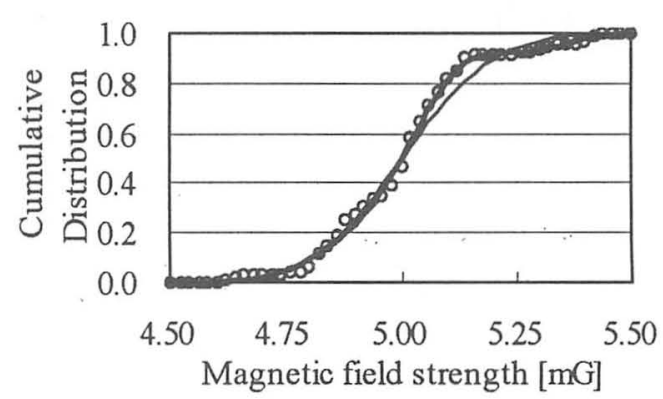

(d) $M=3, N=11$ (based on pulsation)

Fig.5 A comparison between experimentally sampled specific probability distribution (O) and theoretically estimated distribution curves ( - : initial term, higher order approximation) from physiological factors to physical factors.

Figure 5 shows comparisons between experimentally sampled specific probability distribution $P_{s}(y)$ and theoretically estimated distribution curves from physiological factors (i.e. mean blood pressure and pulsation) to physical factors (i.e. sound and magnetic field) in reverse. 
From these figures, it was found that all theoretical curves show a fairly good agreement with experimentally sampled points, in spite of an early stage of study. Hereafter, there remains an important problem to be solved on how higher order expansion parameter $A_{m n}$ is connected concretely with human character.

\section{Conclusions}

In this paper, a stochastic methodology has been discussed first in close connection with the mutual relationship between sound (with other environmental factors) and psychological or physiological factors surrounding an indoor acoustic environment, especially through a system model based on an extended regression analysis reflecting not only the linear but also the nonlinear correlation information. More specifically, in order to mutually predict their probabilistic behavior from one to another, we have proposed a parameter estimation method based on an internal statistical architecture of the joint probability among these factors. Finally, the validity and effectiveness of our proposed method have been confirmed through some principle experiment by applying it to the observation data in the room of an actual living environment.

As mentioned at the beginning, there still remains many unsolved problems in the methodological research in measurements and evaluations which give any suggestion to compound and accumulation effect in the EM environmental problems in an actual working environment. Some future problems to be solved are listed below:

i) find suitable method to be able to gather as many data as possible,

ii) find appropriate method on how to truncate expansion terms based on finite observed data,

iii) improvement of this proposed method considering finite fluctuation range of the observed data.

From now on, it seems to become more important to find out accurate correlation evaluation method and/or accurate measurement technique in a time domain rather than an analytic technique in a frequency domain [17].

\section{ACKNOWLEDGEMENTS}

We would like to express our cordial thanks to the students of the Housing Laboratory of Hiroshima University and the Informational Environmental System Laboratory of Hiroshima Prefectural Women's University for their helpful assistance and discussion.

\section{References}

[1] Measurement Technique Research Committee on Biological Effect of High Frequency Electromagnetic Field, The Institute of Electrical Engineers of Japan Ed., Biological Effect of Electromagnetic Field and Measurement. (Corona-sha, Tokyo, 1995) in Japanese

[2] Measurement Technique Research Committee on Time Domain Electromagnetic Noise, The Institute of Electrical Engineers of Japan Ed., A time domain technique for measurement of the electro- magnetic noise. (Corona-sha, Tokyo, 1995) in Japanese

[3] M. Ohta, A. Ikuta, H. Ogawa, "Mutual relation characteristics among light, sound and electromagnetic waves leaked from the actual working VDT ---Introduction of a system model with nonlinear discrete regression type", J. Acoust. Soc. Jpn., 53, 807-810 (1997)

[4] A. Ikuta, M. Ohta, H. Ogawa, "Various regression characteristics with higher order among light, sound and electromagnetic waves leaked from a VDT ---A measurement and signal processing in the actual working environment", Measurement, J. IMC IMEKO, 21, 25-33 (1997)

[5] M. Ohta, H. Ogawa, "A methodological trial of regression analysis with higher order correlation between electromagnetic and sound waves leaked by a VDT in an actual working environment", J. EM Waves \& Apps., 12, 1357-1367 (1998)

[6] M. Ohta, H. Ogawa, "A trial on hierarchical extraction of higher order correlation between electromagnetic and sound waves around a VDT environment ---Practical use of background noise and probability prediction", in Electromagnetic Waves PIER34, ed. J. A. Kong, Chap.12, 285-298 (EMW Publishing, Cambridge, 2001)

[7] H. Ogawa, M. Ohta, A. Ikuta, "A trial on stochastic evaluation of near-by electromagnetic field leaked from ITE group under parallel operating situation", J. EM Waves \& Apps., 18, 1621-1635 (2004)

[8] H. Ogawa, M. Ohta, H. Iwashige, "Some objective and subjective evaluation methods for room sound, illuminance, temperature and electromagnetic environment", Electronic Journal "Technical Acoustics"<http://ta.org.ru/>, 22 (2005)

[9] M. Ohta, H. Ogawa, A. Ikuta, "Mutual-probability prediction and higher-order correlation effects among acoustic, light, and electromagnetic waves in a video display terminal environment", Comptes Rendus Mecanique, 333, 642-647 (2005)

[10] M. Ohta, A. Ikuta, N. Takaki, "A new trial of regression analysis with less information loss and its application to sound insulation walls ---matching to the prediction of response probability distribution", J. Acoust. Soc. Jpn., 44, 848-856 (1988)

[11] M. Ohta, A. Ikuta, H. Ogawa, "A stochastic evaluation theory in multi-dimensional signal space for EM interference noise and its experimental relationship to acoustic environment", Trans. IEE Jpn. (C), 118, 465-475 (1998)

[12] M. Ohta, Y. Mitani, N. Nakasako, "A fundamental study on the statistical evaluation of receiver response for an electromagnetic wave environment in multi-dimensional signal space---theory and basic experiment", J. EM Waves \& Apps., 12, $677-699$ (1998)

[13] M. Ohta, Y. Mitani, H. Ogawa, "Multi-dimensional generalization in space and time domains for Middleton's study in stochastic evaluation of correlative many EM noise processes", in Electromagnetic Waves PIER24, ed. J. A. Kong, Chap.5, 97-118 (EMW Publishing, Cambridge, 1999)

[14] A. Ikuta, M. Ohta, H. Ogawa, "An Adaptive Signal Processing Method Combining Digital Filter with Fuzzy Inference and Its Application to Wave Motion Type Actual Environment", Trans. IEICE, J82-A, 817-827 (1999) in Japanese

[15] A. Ikuta, M. Ohta, "Two Types of Stochastic Evaluation Method for Electromagnetic Wave in Near Field Leaked from ITE Group under Parallel Working Situation ----Introduction of Compound Law in Space and Time Domain", IEICE Technical Report, EMCJ2000-46, 35-40 (2000) in Japanese

[16] M. Ohta, T. Koizumi, "General statistical treatment of the response of a non-linear rectifying device to a stationary random input", IEEE Trans. IT, IT-14, 595-598 (1968)

[17] Y. Akao, Basis of Environmental Electromagnetic Engineering. (IEICE, Tokyo, 1991) in Japanese 